\title{
Effects of $n-3$ fatty acids, EPA v. DHA, on depressive symptoms, quality of life, memory and executive function in older adults with mild cognitive impairment: a 6-month randomised controlled trial
}

\author{
Natalie Sinn ${ }^{1 *}$, Catherine M. Milte ${ }^{1}$, Steven J. Street ${ }^{2}$, Jonathan D. Buckley ${ }^{1}$, Alison M. Coates ${ }^{1}$, \\ John Petkov ${ }^{3}$ and Peter R. C. Howe ${ }^{1}$ \\ ${ }^{1}$ School of Health Sciences, Sansom Institute for Health Research, Nutritional Physiology Research Centre, University of South \\ Australia, GPO Box 2471, Adelaide, SA 5001, Australia \\ ${ }^{2}$ Institute of Health and Biomedical Innovation, Queensland University of Technology, Brisbane, QLD, Australia \\ ${ }^{3}$ Centre for Regional Engagement, University of South Australia, Adelaide, SA, Australia \\ (Submitted 22 March 2011 - Final revision received 27 July 2011 - Accepted 27 July 2011 - First published online 20 September 2011)
}

\begin{abstract}
Depressive symptoms may increase the risk of progressing from mild cognitive impairment (MCI) to dementia. Consumption of $n$ - 3 PUFA may alleviate both cognitive decline and depression. The aim of the present study was to investigate the benefits of supplementing a diet with $n$-3 PUFA, DHA and EPA, for depressive symptoms, quality of life (QOL) and cognition in elderly people with MCI. We conducted a 6-month double-blind, randomised controlled trial. A total of fifty people aged $>65$ years with MCI were allocated to receive a supplement rich in EPA (1.67 g EPA + 0.16 g DHA/d; $n$ 17), DHA (1.55 g DHA + 0.40 g EPA/d; $n$ 18) or the $n-6$ PUFA linoleic acid (LA; $2 \cdot 2 \mathrm{~g} / \mathrm{d} ; n$ 15). Treatment allocation was by minimisation based on age, sex and depressive symptoms (Geriatric Depression Scale, GDS). Physiological and cognitive assessments, questionnaires and fatty acid composition of erythrocytes were obtained at baseline and 6 months (completers: $n$ 40; EPA $n$ 13, DHA $n$ 16, LA $n$ 11). Compared with the LA group, GDS scores improved in the EPA $(P=0 \cdot 04)$ and DHA $(P=0 \cdot 01)$ groups and verbal fluency (Initial Letter Fluency) in the DHA group $(P=0 \cdot 04)$. Improved GDS scores were correlated with increased DHA plus EPA ( $r$ 0.39, $P=0 \cdot 02$ ). Improved self-reported physical health was associated with increased DHA. There were no treatment effects on other cognitive or QOL parameters. Increased intakes of DHA and EPA benefited mental health in older people with MCI. Increasing $n$-3 PUFA intakes may reduce depressive symptoms and the risk of progressing to dementia. This needs to be investigated in larger, depressed samples with MCI.
\end{abstract}

Key words: Mild cognitive impairment: Dementia: Depression: $n$-3 Fatty acids: EPA: DHA

Dementia, the most common form being Alzheimer's disease, is characterised by progressive and profound loss of memory, cognitive function and ability to carry out daily functional activities of living. It has been associated with various genetic and environmental risk factors ${ }^{(1)}$. Its prevalence is increasing rapidly worldwide ${ }^{(2)}$ and it represents a major public health concern. Therefore, any delay in its onset will have significant social and economic impacts, making early intervention an international imperative. It has been suggested that mild cognitive impairment (MCI) is possibly the earliest stage of detectable dementia ${ }^{(3)}$ and may be the optimal time to intervene with preventive therapies ${ }^{(4)}$. Furthermore, major and minor depression often occurs in patients with $\mathrm{MCI}^{(5)}$, and this increases the risk of progressing to dementia ${ }^{(6)}$.
In Western populations, there has been a marked decrease in the dietary intake of $n$-3 PUFA along with a substantial increase in the consumption of $n-6 \mathrm{PUFA}^{(7)}$. The long-chain $n-3$ PUFA DHA (22:6n-3) is highly concentrated in the brain and has been associated with numerous structural and functional roles ${ }^{(8-10)}$. The long-chain $n-3$ PUFA EPA $(22: 5 n-3)$ is also thought to have important functions in the brain ${ }^{(11)}$ via the synthesis of benign eicosanoids that tend to counter the inflammatory, thrombotic and primarily vasoconstrictor properties of eicosanoids produced from the $n-6$ PUFA arachidonic acid (AA; $20: 4 n-6)^{(12)}$.

With ageing, neural membrane fluidity is compromised due to the increased presence of cholesterol, the reduced activity of desaturase enzymes, blockages to phospholipid pathways

Abbreviations: AA, arachidonic acid; GDS, Geriatric Depression Scale; LA, linoleic acid; MCI, mild cognitive impairment; WAIS III, Wechsler Adult Intelligence Scale. 
and increased oxidative stress ${ }^{(13)}$, all of which are inversely associated with PUFA. Brain autopsies of Alzheimer's disease patients have shown significantly higher saturated fat and lower $n$-3 PUFA content in the hippocampus and frontal lobes (governing memory and executive function, respectively) compared with age-matched controls ${ }^{(14)}$, consistent with reports of decreased hippocampus size and function in Alzheimer's disease patients ${ }^{(13)}$.

To date, few clinical trials evaluating the benefits of $n-3$ PUFA in patients with dementia have been published, although more are under way ${ }^{(15)}$. There are some reports of benefit ${ }^{(16,17)}$. However, larger trials indicate that people with MCI are more likely to respond. These studies investigated supplementation of $n-3$ PUFA in patients with dementia but only found improvements in the subgroups with mild cognitive decline ${ }^{(18-20)}$. In elderly, cognitively healthy populations, there does not appear to be benefit ${ }^{(21,22)}$; however, a recent study did report improvements following DHA supplementation in elderly people with age-related cognitive decline $^{(23)}$. This body of studies supports indications that intervention with $n$-3 PUFA may be more effective in early stages of cognitive decline, yet no studies have yet focused exclusively on people with MCI and therefore possibly in the early stages of dementia onset.

Depression is very common in older adults with MCI and increases the risk of developing dementia ${ }^{(6)}$. Clinical trials investigating the efficacy of $n-3$ supplementation in alleviating depressive symptoms have found positive results in about half of published studies ${ }^{(15,24)}$. Among other methodological differences, studies have used varying ratios of EPA:DHA; some have used only DHA or ethyl-EPA.

The purpose of the present study was to compare the effects of high-EPA and high-DHA fish oils with n-6 PUFA linoleic acid (LA; 18:2n-6), a vegetable oil, on depressive symptoms, quality of life, memory and executive function in elderly people with MCI, to investigate the associations between increased erythrocyte DHA and EPA levels and any improved symptoms, and to determine whether those with lower baseline erythrocyte PUFA levels respond more readily to supplementation.

\section{Experimental methods}

\section{Participants and procedure}

Eligibility criteria considered discussions by the International Working Group on $\mathrm{MCI}^{(3,25)}$. Identification of MCI is generally defined as signs of cognitive decline beyond those expected for age but not dementia. Therefore, subjective complaints of memory loss but otherwise normal daily functioning are recommended, along with cognitive assessment scores $\geq 1.5 \mathrm{sD}$ below the population mean. We screened elderly people aged over 65 years who had self-reported memory loss, were able to maintain normal daily functioning and gave informed consent, did not eat fish more than once per week, and had not taken any fish oil supplements within the previous 3 months. They had to be prepared to attend a screening, complete two rounds of assessments, take daily supplements, keep a weekly diary of fish intake and not to consume fish more than once per fortnight during the study. Those who met the following criteria after screening were invited to take part: standardised Mini-Mental State Examination scores $\geq 22$ (a score $\leq 20$ indicates possible dementia), recall memory (Verbal Paired Associates Task $^{(26)}$ ) age-standardised scores $\geq 1.5 \mathrm{sD}$ below the population mean and/or age- and education-adjusted DemTect scores between 9 and $12^{(27)}$ (these tests are described further below).

According to Cohen's $f$, the required $n$ to detect a medium effect size $(d=0 \cdot 50)$ in a repeated-measures within-between interaction design for three groups with a power of 0.80 is sixty-six participants (twenty-two per group). Recruitment took place in Adelaide and Brisbane, Australia, via newspaper, magazine and radio advertisements, retirement villages (talks and letterbox drops), Alzheimer's Association, shopping malls and television. A flow chart of participants through the study is depicted in Fig. 1. After 6 months of extensive screening, fifty eligible volunteers were recruited: forty-four in Adelaide and six in Brisbane (see Table 1 for baseline characteristics), of whom forty completed the 6-month assessments. This provided adequate power to detect an effect size of $d=0.64$, or $d=0.56$ with all cases ( $n$ 50) included in the analyses (assuming a balanced dataset). The intervention took place between March 2009 and March 2010.

As participants were enrolled, they were independently allocated to one of three conditions (EPA-rich oil, DHArich oil or placebo) using the process of minimisation based on age, Geriatric Depression Scale (GDS) scores and sex, respectively. Altman \& Bland $^{(28)}$ recommended this simple process for small trials with progressive enrolment in order to achieve balanced treatment groups without investigator bias. Of the participants, eighteen (36\%) had GDS scores of 4 or above, indicating possible depression. All researchers involved with participants, data entry or analysis and participants were blinded to treatment conditions. Supplements were coded and labelled independently; participants were asked at 6 months which condition they thought they were in (and why) and these observations were recorded.

Eligible volunteers completed assessments at baseline and 6 months at the University of South Australia (Adelaide, SA, Australia) or the Institute of Health and Biomedical Innovation (Brisbane, QLD, Australia) in the morning following an overnight fast. Height, weight and blood pressure were measured and blood samples collected. Volunteers were then offered breakfast (toast, cereal, tea, orange juice and water) and underwent $45 \mathrm{~min}$ of cognitive assessments. The same instructions and protocol were used for each volunteer's assessments.

The project was performed in accordance with the ethical standards laid down in the 1964 Declaration of Helsinki, and all procedures involving human participants were approved by the Human Research Ethics Committees at the University of South Australia and Queensland University 
of Technology. Informed written consent was obtained from all participants.

\section{Supplements}

Participants were randomly allocated to EPA-rich fish oil, providing $1.67 \mathrm{~g} \mathrm{EPA}+0.16 \mathrm{~g} \mathrm{DHA} / \mathrm{d}$, DHA-rich fish oil, providing $1.55 \mathrm{~g} \mathrm{DHA}+0.40 \mathrm{~g} \mathrm{EPA} / \mathrm{d}$, or control (safflower oil), providing $2 \cdot 2 \mathrm{~g}$ LA ( $n-6$ PUFA)/d for 6 months. The oils were taken in four capsules daily. Participants were asked to return all of their jars at the end of the study and capsules were counted to assess compliance. Adverse events were recorded on the 6-month questionnaires and via regular phone calls throughout the study.

\section{Assessment tools}

All of the following assessments were conducted at baseline and 6 months.

Depression and quality of life

Geriatric depression scale. The fifteen-item short form of the GDS $^{(29)}$ was our primary outcome measure of depressive symptoms. Respondents circle 'yes' or 'no' in response to a series of statements, e.g. 'Are you in good spirits most of the time', 'Do you feel that your life is empty'. A score of one point is allocated for each response that is indicative of possible depression. Therefore, a higher score indicates greater symptom severity; scores of 4 or above possible depression. The highest possible score is 15 .

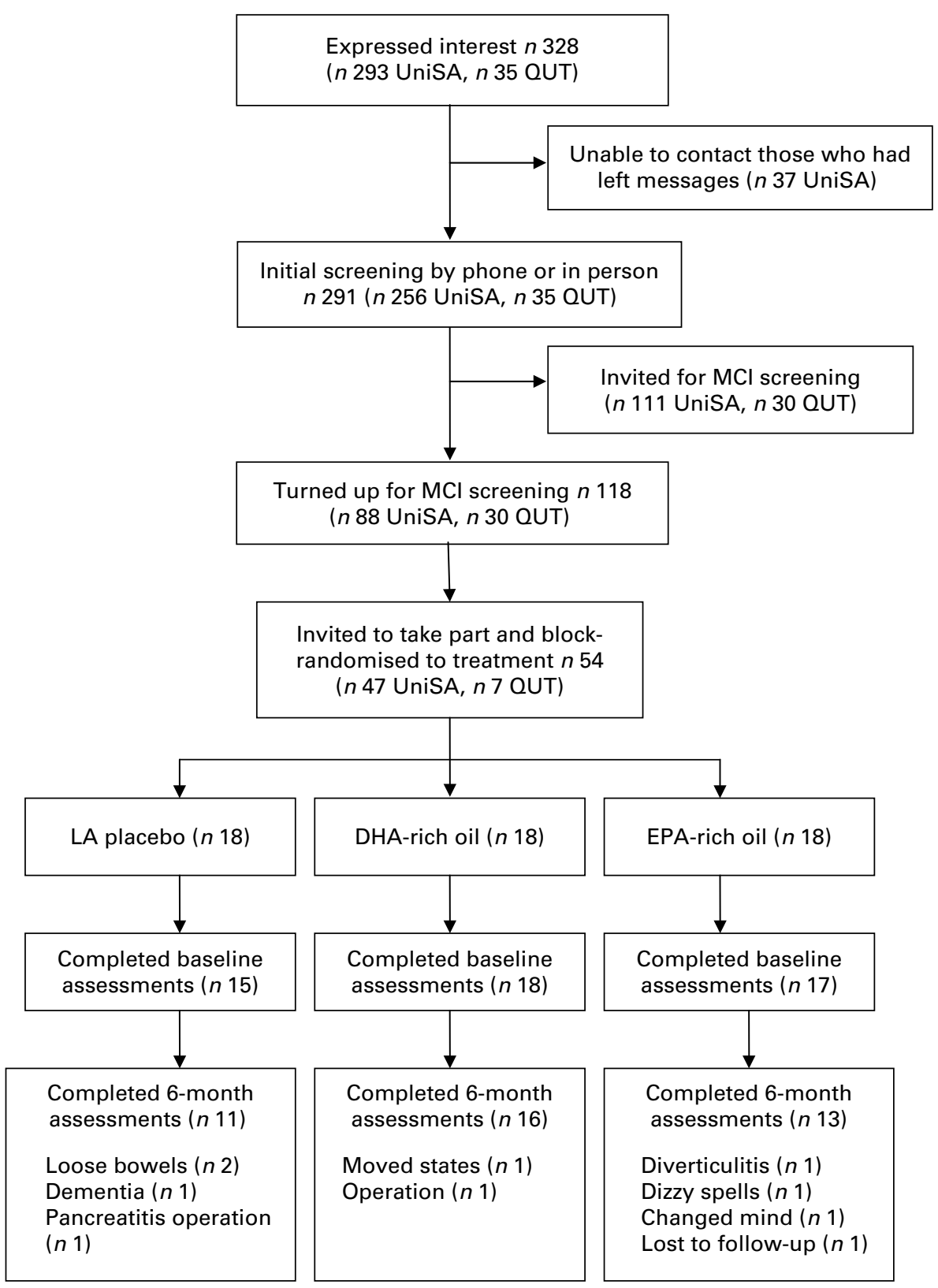

Fig. 1. Flow of participants through the study. UniSA, University of South Australia; QUT, Queensland University of Technology; MCI, mild cognitive impairment; LA, linoleic acid. 
SF-36 health survey. This survey assesses eight domains of health and quality of life: physical functioning, limitations in usual role due to physical health problems (role - physical), bodily pain, general health, vitality, social functioning, limitations in usual role due to emotional problems (role emotional) and mental health. By combining these domains, two further subscales are derived: physical health and mental health. We used the Australian adaptation ${ }^{(30)}$. Where necessary, scales are reverse-scored so that on all the scales, a higher score indicates better health. Each subscale varies in the length of items; each item response is given from a selection varying from 'excellent' to 'poor', 'yes, limited a lot' to 'no, not limited at all', 'all of the time' to 'none of the time', and so on, depending on the nature of the question.

Cognition. Subjective and objective tests were selected that can predict memory loss or dementia and may therefore be sensitive to meaningful improvements in or alleviations of cognitive decline. We selected specific subtests that may be sensitive to the effects of the intervention on memory and executive function and have been used previously in drug trials for $\mathrm{MCI}^{(31)}$. Where necessary, alternate versions of the tests were used for re-test purposes.

Table 1. Baseline sample characteristics $(n 50)^{*}$

(Mean values, standard deviations, number of participants and percentages)

\begin{tabular}{|c|c|c|c|c|c|c|}
\hline & \multicolumn{2}{|c|}{ Control (LA, $n$ 15) } & \multicolumn{2}{|c|}{ DHA-rich oil $(n 18)$} & \multicolumn{2}{|c|}{ EPA-rich oil $(n 17)$} \\
\hline & Mean & SD & Mean & SD & Mean & SD \\
\hline Age (years) & 73 & 3.96 & $74 \cdot 22$ & $7 \cdot 00$ & 74.88 & 5.06 \\
\hline \multicolumn{7}{|l|}{ Sex (male) } \\
\hline$n$ & \multirow{2}{*}{\multicolumn{2}{|c|}{$\begin{array}{c}7 \\
47\end{array}$}} & \multicolumn{2}{|c|}{$\begin{array}{l}13 \\
72\end{array}$} & \multicolumn{2}{|c|}{14} \\
\hline$\%$ & & & \multicolumn{2}{|c|}{72} & \multicolumn{2}{|c|}{82} \\
\hline Highest level of education attained $\dagger$ & $3 \cdot 14$ & 1.88 & $2 \cdot 56$ & 1.98 & $2 \cdot 67$ & 1.50 \\
\hline Family history of dementia or $A D$ & & & & & & \\
\hline$n$ & \multicolumn{2}{|c|}{5} & \multicolumn{2}{|c|}{3} & \multicolumn{2}{|c|}{6} \\
\hline$\%$ & \multicolumn{2}{|c|}{33} & \multicolumn{2}{|c|}{17} & \multicolumn{2}{|c|}{35} \\
\hline Baseline MMSE score & $27 \cdot 73$ & $2 \cdot 22$ & $27 \cdot 83$ & 1.92 & 25.94 & $3 \cdot 31$ \\
\hline Baseline VPA score & $6 \cdot 87$ & 3.07 & $6 \cdot 22$ & 2.53 & $6 \cdot 31$ & $2 \cdot 12$ \\
\hline Baseline DemTect score & $13 \cdot 87$ & $3 \cdot 16$ & $13 \cdot 22$ & 3.41 & $12 \cdot 18$ & 2.63 \\
\hline Baseline GDS score & $3 \cdot 15$ & $2 \cdot 08$ & $3 \cdot 19$ & $3 \cdot 17$ & 4.40 & 2.92 \\
\hline BMI $\left(\mathrm{kg} / \mathrm{m}^{2}\right)$ & $28 \cdot 11$ & $5 \cdot 27$ & $26 \cdot 80$ & 2.58 & 28.05 & 4.07 \\
\hline Systolic blood pressure $(\mathrm{mmHg})$ & $131 \cdot 13$ & 18.51 & $136 \cdot 52$ & $20 \cdot 07$ & 139.02 & $19 \cdot 69$ \\
\hline Diastolic blood pressure $(\mathrm{mmHg})$ & $76 \cdot 33$ & $10 \cdot 46$ & 79.83 & 9.91 & $80 \cdot 10$ & 9.49 \\
\hline Resting heart rate (bpm) & $55 \cdot 33$ & $10 \cdot 96$ & 64.08 & $12 \cdot 54$ & 58.93 & $8 \cdot 14$ \\
\hline \multicolumn{7}{|l|}{ Diabetes } \\
\hline$n$ & \multicolumn{2}{|c|}{2} & \multicolumn{2}{|c|}{4} & \multicolumn{2}{|c|}{1} \\
\hline$\%$ & \multicolumn{2}{|c|}{13} & \multicolumn{2}{|c|}{22} & \multicolumn{2}{|c|}{6} \\
\hline \multicolumn{7}{|l|}{ Current smokerł } \\
\hline$n$ & & & & & & \\
\hline$\%$ & & & & & & \\
\hline Excess alcohol§ & $2 \cdot 33$ & $1 \cdot 18$ & $2 \cdot 22$ & 1.22 & $2 \cdot 38$ & 1.41 \\
\hline SF-36 physical functioning & $23 \cdot 71$ & 4.88 & 24.94 & $3 \cdot 80$ & 24.00 & 4.83 \\
\hline SF-36 vitality & $15 \cdot 60$ & 3.23 & $17 \cdot 29$ & 3.00 & 16.44 & 3.74 \\
\hline SF-36 bodily pain & 8.00 & 1.73 & 8.29 & 1.99 & 8.41 & 2.40 \\
\hline SF-36 physical health & 63.82 & 11.62 & $66 \cdot 81$ & 9.91 & $67 \cdot 15$ & $11 \cdot 20$ \\
\hline SF-36 role - physical & 14.40 & 4.58 & 15.50 & $2 \cdot 73$ & 14.75 & 3.98 \\
\hline SF-36 role - emotional & $11 \cdot 29$ & $3 \cdot 100$ & 11.06 & $2 \cdot 22$ & 11.00 & 3.08 \\
\hline SF-36 mental health & $58 \cdot 79$ & $10 \cdot 81$ & 24.53 & 3.68 & 58.94 & 11.69 \\
\hline RAVLT, total immediate recall & 37.43 & 6.93 & 34.78 & $9 \cdot 00$ & 30.41 & 8.51 \\
\hline RAVLT, immediate delayed recall & $6 \cdot 00$ & 3.23 & 5.89 & 2.95 & 4.94 & 3.03 \\
\hline RAVLT, 20 min delayed recall & 9.79 & 3.02 & $5 \cdot 39$ & $3 \cdot 81$ & $8 \cdot 76$ & 3.29 \\
\hline Digits Forward score & $9 \cdot 60$ & 1.84 & $9 \cdot 28$ & 1.99 & $9 \cdot 88$ & $2 \cdot 12$ \\
\hline Boston Naming Task & 51.53 & $6 \cdot 32$ & 53.94 & $6 \cdot 77$ & 51.47 & 5.52 \\
\hline Letter-Number Sequencing & 8.40 & 2.03 & 7.00 & $2 \cdot 61$ & 6.53 & 2.48 \\
\hline Digits Backward score & $5 \cdot 87$ & 1.60 & 6.06 & 1.39 & $5 \cdot 35$ & 1.41 \\
\hline Trail-Making score & 3.00 & $1 \cdot 27$ & 3.34 & $1 \cdot 28$ & 3.43 & 1.50 \\
\hline Stroop Colour-Word Test score & 2.94 & 0.70 & 3.06 & 0.78 & $3 \cdot 20$ & $1 \cdot 17$ \\
\hline Initial Letter Fluency total & $23 \cdot 60$ & $8 \cdot 18$ & $21 \cdot 39$ & $7 \cdot 14$ & $18 \cdot 24$ & 8.45 \\
\hline Excluded Letter Fluency total & $15 \cdot 67$ & 6.43 & 14.50 & $6 \cdot 84$ & 11.59 & 4.45 \\
\hline
\end{tabular}

LA, linoleic acid; AD, Alzheimer's disease; MMSE, Mini-Mental State Examination; VPA, Verbal Paired Associates (age- and sex-adjusted standardised score, $M=10$ ); GDS, Geriatric Depression Scale (scores $>4$ may indicate clinical depression); bpm, beats per minute; RAVLT, Rey Auditory Verbal Learning Test

* Mean values were not significantly different between the groups for any of these variables (one-way ANOVA or $\chi^{2}$ tests).

† Education: 1, years 8-10; 2, year 11; 3, year 12; 4, technical and further education (or 13-14 years of total education); 5 , university; 6 , postgraduate.

$\ddagger$ Current smoker: 'yes/no'.

$\S$ Frequency of consuming more than two alcoholic drinks per d (1, never/rarely; 2, occasionally; 3, once per week; 4, few days per week; 5, daily). The SF-36 scales are reverse-scored, where necessary, so that a higher score always represents improved health; each scale has a different scale and number of items and therefore different possible total scores. 


\section{Memory}

Memory Functioning Questionnaire. This questionnaire ${ }^{(32)}$ assesses four domains of subjective perception of memory loss in the recent and more distant past: general frequency of forgetting, seriousness of forgetting, retrospective functioning and mnemonics usage.

Rey Auditory Verbal Learning Test. This test ${ }^{(33)}$ measures immediate, delayed recall and recognition memory ${ }^{(32)}$. It requires participants to recall a list of fifteen words learned over five trials, immediately, then after a distracter list and again $20 \mathrm{~min}$ later. To test recognition memory, they are then given a list of words containing the target words and asked to select those that were on the list. The immediate memory score is calculated by adding trials $1-5$ together (total 75), the delayed recall by totalling the number of words recalled on that trial (total 15) and recognition memory by totalling the number of words correctly recognised (total 15).

Digits Forward. It is a subtest from the Wechsler Adult Intelligence Scale (WAIS III) ${ }^{(34)}$ that requires participants to recall a random series of numbers starting with 2 and increasing to 9. The task is discontinued following failure on both items of the same length. The maximum score that can be obtained is 16 (two on each trial).

Boston Naming Task. This task ${ }^{(33)}$ tests verbal expression and may predict dementia ${ }^{(35)}$. It consists of sixty large pictures of items ranging in familiarity from common items such as 'pencil' to less common objects such as 'sphinx' and is discontinued after eight consecutive failures. A score of one point is given for each correct response; the maximum score that can be obtained is 60 .

\section{Executive function/working memory}

Letter-Number Sequencing. It is a subtest of the WAIS III ${ }^{(33)}$ that measures working memory and executive function. It requires participants to recall strings of digits and letters, reorganising them so that digits are recalled first in numerical order and then letters in alphabetical order, starting with two and increasing up to eight consecutive letters/numbers with two trials of each. It is discontinued following failure on both items of the same length. The maximum score that can be obtained is 14 (two on each trial).

Digits Backward. It is another subtest from the WAIS III that measures working memory and executive function ${ }^{(34)}$. Following Digits Forward, participants are asked to recall number sequences in reverse order, starting with two and increasing to eight numbers and discontinued following failure on both items of each trial. The maximum score that can be obtained is 14 (two on each trial).

Trail-Making Task. This task ${ }^{(33)}$ is a measure of scanning and visuomotor tracking, divided attention, and cognitive flexibility. It requires volunteers to draw lines as quickly as they can to connect consecutively numbered circles on one worksheet (part A) and then connect consecutively numbered and lettered circles on another worksheet by alternating between the two sequences (part B). The score is derived by dividing the time taken (in s) during part $\mathrm{B}$ by the time taken during part A.
Stroop Colour-Word Test. This test ${ }^{(36)}$ measures the ability to ignore distracting information. Part A requires participants to read a sheet of colour names. In part $\mathrm{B}$, they are asked to name the colours (which are incongruent with the words; for example, the word blue may be printed in the colour red) and ignore the words. The score is derived by dividing the time taken (in s) during part B by the time taken during part A.

Verbal fluency. Strategic retrieval of verbal material was assessed by tests of initial and excluded letter fluency ${ }^{(37,38)}$. These tests require participants to produce as many words as possible within $60 \mathrm{~s}$ either beginning with a designated letter (e.g. F, S) or not containing a designated letter (e.g. E, A), respectively. A score of one point is given for each correct word that is generated within $60 \mathrm{~s}$ for each respective trial.

Potential confounders. A background questionnaire assessed potential confounders: highest level of education, frequency of consuming more than two alcoholic drinks/d, whether or not they smoke, medications, medical conditions and family history of dementia (see Table 1).

Physiological parameters. We tested heart rate, BMI and blood pressure as potential confounders. Height and weight were measured by a stadiometer (Seca, Hamburg, Germany) and scales (Tanita, Tokyo, Japan), respectively. Blood samples were collected into $6 \mathrm{ml} \mathrm{K}$ EDTA tubes, $5 \mathrm{ml}$ serum tubes and $4 \mathrm{ml}$ lithium heparin tubes (Interpath Services Pty Ltd, Heidelberg West, VIC, Australia) by venepuncture. Following the blood sample, volunteers were invited to rest quietly in a darkened room for a minimum of $10 \mathrm{~min}$ before three measures of blood pressure and heart rate were taken using an automatic blood pressure monitor (model IA1B; Omron, Kyto, Japan), with 5 min rest between each measure.

Assessment of fatty acid profiles. Relative proportions of individual fatty acids in erythrocytes were assessed using a method adapted from previously established methods ${ }^{(39-41)}$. Erythrocytes were isolated within $2 \mathrm{~h}$ of collection by centrifugation, washed in isotonic saline and stored at $-80^{\circ} \mathrm{C}$. They were subsequently thawed and the lipids were extracted with chloroform and isopropanol (2:1). The organic phase containing the lipid was evaporated to dryness under a stream of $\mathrm{N}_{2}$ gas. The lipids were then transesterified with acetyl chloride in methanol toluene $(4: 1, \mathrm{v} / \mathrm{v})$ at $100^{\circ} \mathrm{C}$ for $1 \mathrm{~h}$. The resultant fatty acid methyl esters were extracted with $10 \%$ potassium carbonate. Fatty acid methyl esters were separated and quantified using a Shimadzu 2010 gas chromatograph equipped with a $50 \mathrm{~m}$ capillary column $(0.32 \mathrm{~mm}$, inner diameter $)$ coated with BPX-70 (0.25 $\mu \mathrm{m}$ film thickness; SGE Analytical Science Pty Limited, Ringwood, VIC, Australia). The injector temperature was set at $250^{\circ} \mathrm{C}$ and the detector (flame ionisation) temperature at $260^{\circ} \mathrm{C}$. The initial oven temperature was $130^{\circ} \mathrm{C}$ and was programmed to rise to $220^{\circ} \mathrm{C}$ at $5^{\circ} \mathrm{C} / \mathrm{min} . \mathrm{H}_{2}$ was used as the carrier gas at a velocity of $36.4 \mathrm{~cm} / \mathrm{s}$. Fatty acid methyl esters were identified based on the retention time to authentic lipid standards (GLC-463; Nu-Chek Prep, Inc., Elysian, MN, USA). 


\section{Statistical analysis}

Data analysis was conducted using SPSS Statistics (version 17.0; SPSS, Inc., Chicago, IL, USA). Baseline one-way ANOVA were used for parametric data and $\chi^{2}$ analyses for non-parametric data to examine whether demographic, physiological and dependent variables (mood, quality of life and cognition) were evenly distributed between the groups. Linear mixed model analyses were planned to investigate the effects of DHA and EPA $v$. LA on outcome variables (assuming data were missing at random). Regressions were used to investigate the correlations between increased PUFA levels and improved outcomes and baseline PUFA levels with improved outcomes. Due to the relatively small sample size, $P$ values were set at 0.05 to avoid the possibility of a type II error.

\section{Results}

\section{Adverse events}

The treatments were well tolerated apart from a few gastrointestinal-related complaints. From the LA (control) group, three complained of bowel problems, one of nausea and one had reflux. From the EPA-rich oil group, there was one case reported for each of the following: flatulence, nausea, reflux and upset stomach, and one participant withdrew due to diverticulitis. No adverse events were reported in the DHA group.

In the LA, DHA and EPA groups, one, eight and six persons, respectively, guessed that they had been taking fish oil
$(P=0 \cdot 09)$; six, three and two individuals, respectively, guessed they were on placebo and two, five and three did not know. However, of the people who guessed the condition they were in, whether correctly or not, one, three and five from each group said it was because of a fishy taste; seven, seven and three said it was because they thought they were either not deriving or were deriving benefits (mostly physical, e.g. less aches and pains) from the treatment. Therefore, treatment blinding was successful on the whole.

\section{Compliance}

Capsule counts indicated excellent compliance, with an average supplement consumption of $93 \%$ across the groups (DHA 97\%, EPA 94\% and LA 82\%). This is confirmed by changes in erythrocyte $n-3$ and $n$ - 6 PUFA levels after 6 months for each treatment group (see Table 2). As shown in Table 2, PUFA levels corresponded to the respective treatments (e.g. DHA levels increased in the DHA treatment group, etc.).

\section{Primary analysis: effect of the supplement}

There were eleven missing observations in the follow-up data; however, demographic data were complete. Logistic regression, with missingness as the dependent variable and demographic variables (sex, age, condition, history of dementia and Alzheimer's disease) as predictors, showed that none of the predictors were significant in predicting missingness, and the number of dropouts between the

Table 2. Erythrocyte PUFA levels at baseline and 6 months in each treatment group (as percentage of fatty acids) ${ }^{*}$

(Mean values and standard deviations)

\begin{tabular}{|c|c|c|c|c|c|}
\hline & \multicolumn{2}{|c|}{ Baseline } & \multicolumn{2}{|c|}{6 months } & \multirow{2}{*}{$\begin{array}{c}\text { Change in } \\
P \text { value }\end{array}$} \\
\hline & Mean & SD & Mean & SD & \\
\hline \multicolumn{6}{|c|}{ LA control group $(n 15, n 11)$} \\
\hline EPA & 0.95 & 0.37 & 0.79 & 0.17 & 0.127 \\
\hline DHA & 4.74 & 1.00 & 4.58 & $1 \cdot 13$ & 0.096 \\
\hline Total $n-3$ PUFA & 8.40 & 1.55 & $8 \cdot 16$ & 1.43 & 0.270 \\
\hline LA & $8 \cdot 20$ & 1.61 & $7 \cdot 24$ & 0.88 & 0.072 \\
\hline AA & 11.39 & 1.17 & $12 \cdot 08$ & 0.77 & 0.171 \\
\hline Total $n-6$ PUFA & 24.19 & 1.65 & $24 \cdot 38$ & 1.40 & 0.886 \\
\hline \multicolumn{6}{|c|}{ DHA group $(n 18, n 16)$} \\
\hline EPA & 0.97 & 0.25 & 1.83 & 0.38 & 0.000 \\
\hline DHA & 4.55 & 0.68 & 8.65 & 0.83 & 0.000 \\
\hline Total $n-3$ PUFA & $8 \cdot 21$ & 1.03 & 12.85 & 1.27 & 0.000 \\
\hline LA & $7 \cdot 80$ & $1 \cdot 24$ & 6.00 & 0.61 & 0.000 \\
\hline AA & 11.64 & 1.48 & $10 \cdot 22$ & 1.52 & 0.000 \\
\hline Total $n-6$ PUFA & 24.07 & $1 \cdot 27$ & $19 \cdot 69$ & 1.37 & 0.000 \\
\hline \multicolumn{6}{|c|}{ EPA group $(n 17, n 12)$} \\
\hline EPA & 0.96 & 0.23 & 4.06 & 0.76 & 0.000 \\
\hline DHA & 4.56 & 0.81 & $5 \cdot 34$ & 0.65 & 0.002 \\
\hline Total $n-3$ PUFA & $8 \cdot 31$ & 1.00 & 14.35 & 1.56 & 0.000 \\
\hline LA & $7 \cdot 80$ & $(1 \cdot 36)$ & 5.66 & $(0.97)$ & 0.000 \\
\hline AA & 11.53 & $(0.91)$ & 9.49 & $(0.96)$ & 0.000 \\
\hline Total $n-6$ PUFA & 23.91 & $(1.37)$ & $18 \cdot 14$ & $(1 \cdot 19)$ & 0.000 \\
\hline
\end{tabular}

LA, linoleic acid; AA, arachidonic acid.

* Mean values were not significantly different between the groups for baseline PUFA levels (one-way ANOVA).

† Changes from baseline to 6 months were analysed using paired-samples $t$ tests. 
Table 3. Treatment effects (time $\times$ treatment interactions) for linear mixed model analysis of all cases as randomised to treatment (EPA $v$. linoleic acid (LA), DHA $v$. LA) on depression, health-related quality of life and cognitive assessments over 6 months ( $n$ 50; LA $n$ 15, DHA $n$ 18, EPA $n$ 17)

(Estimates, standard errors and $95 \%$ confidence intervals)

\begin{tabular}{|c|c|c|c|c|c|c|}
\hline & Estimate & SE & $t$ score & $P$ & $95 \% \mathrm{Cl}$ & ICC†‡ \\
\hline \multicolumn{7}{|l|}{ Depression and quality of life } \\
\hline Geriatric depression scale & & & & & & 0.87 \\
\hline EPA $v$. LA & -1.23 & 0.56 & $-2 \cdot 18$ & $0.04^{*}$ & $-2.37,-0.09$ & \\
\hline DHA v. LA & -1.40 & 0.53 & -2.62 & $0.01^{*}$ & $-2.47,-0.32$ & \\
\hline SF-36 physical functioning & & & & & & 0.82 \\
\hline EPA $v$. LA & -0.02 & $1 \cdot 12$ & -0.02 & 0.98 & $-2 \cdot 30,2 \cdot 25$ & \\
\hline DHA v. LA & 1.30 & 1.05 & 1.23 & 0.23 & $-0.84,3.43$ & \\
\hline SF-36 vitality & & & & & & 0.73 \\
\hline EPA $v$. LA & 0.73 & 1.01 & 0.72 & 0.47 & $-1 \cdot 32,2 \cdot 79$ & \\
\hline DHA v. LA & 1.57 & 0.94 & 1.68 & $0 \cdot 10$ & $-0.33,3.47$ & \\
\hline SF-36 bodily pain & & & & & & 0.52 \\
\hline EPA v. LA & 0.19 & 0.85 & 0.22 & 0.83 & $-1.53,1.91$ & \\
\hline DHA v. LA & 0.83 & $0 \cdot 81$ & 1.03 & 0.31 & $-0.80,2.47$ & \\
\hline SF-36 physical health & & & & & & 0.77 \\
\hline EPA v. LA & -1.44 & $3 \cdot 12$ & -0.46 & 0.65 & $-7.78,4.90$ & \\
\hline DHA v. LA & 3.73 & $2 \cdot 86$ & 1.31 & 0.20 & $-2.07,9.54$ & \\
\hline SF-36 role - physical & & & & & & 0.57 \\
\hline EPA $v$. LA & 0.09 & 1.36 & 0.07 & 0.95 & $-2 \cdot 66,2 \cdot 85$ & \\
\hline DHA v. LA & 0.74 & $1 \cdot 30$ & 0.57 & 0.57 & $-1.89,3.38$ & \\
\hline SF-36 role - emotional & & & & & & 0.67 \\
\hline EPA $v$. LA & $0 \cdot 10$ & 1.00 & 0.10 & 0.92 & $-1.92,2 \cdot 13$ & \\
\hline DHA v. LA & 0.31 & 0.93 & 0.34 & 0.74 & $-1.57,2 \cdot 20$ & \\
\hline SF-36 mental health & & & & & & 0.77 \\
\hline EPA $v$. LA & 5.43 & 3.09 & 1.76 & 0.09 & $-0.83,11.69$ & \\
\hline DHA v. LA & 3.29 & $2 \cdot 73$ & 1.20 & 0.24 & $-2 \cdot 26,8 \cdot 83$ & \\
\hline \multicolumn{7}{|l|}{ Cognitive assessments } \\
\hline RAVLT, total & & & & & & 0.56 \\
\hline EPA $v$. LA & -0.75 & $3 \cdot 16$ & -0.24 & 0.81 & $-7 \cdot 14,5 \cdot 65$ & \\
\hline DHA v. LA & $-2 \cdot 28$ & 3.00 & -0.76 & 0.45 & $-8.35,3.79$ & \\
\hline RAVLT, delayed recall & & & & & & 0.79 \\
\hline EPA $v$. LA & 0.53 & 0.93 & 0.57 & 0.57 & $-1.35,2.42$ & \\
\hline DHA v. LA & 0.36 & 0.88 & 0.41 & 0.69 & $-1 \cdot 43,2 \cdot 14$ & \\
\hline RAVLT, recognition & & & & & & 0.51 \\
\hline EPA $v$. LA & 0.56 & 1.38 & 0.41 & 0.69 & $-2 \cdot 23,3.35$ & \\
\hline DHA v. LA & 0.02 & 1.31 & 0.02 & 0.99 & $-2.63,2.67$ & \\
\hline Digits Forward & & & & & & 0.50 \\
\hline EPA v. LA & -0.62 & 0.84 & -0.74 & 0.47 & $-2.32,1.08$ & \\
\hline DHA v. LA & 0.12 & 0.80 & 0.15 & 0.88 & $-1.49,1.73$ & \\
\hline Boston Naming Task & & & & & & 0.80 \\
\hline EPA $v$. LA & -1.68 & $1 \cdot 34$ & -1.25 & 0.22 & $-4.41,1.05$ & \\
\hline DHA v. LA & -1.56 & $1 \cdot 24$ & -1.26 & 0.22 & $-4.07,0.96$ & \\
\hline Letter-Number Sequencing & & & & & & 0.36 \\
\hline EPA $v$. LA & 0.22 & 0.81 & 0.27 & 0.79 & $-1.43,1.87$ & \\
\hline DHA v. LA & 0.12 & 0.77 & 0.15 & 0.88 & $-1.44,1.68$ & \\
\hline Digits Backward & & & & & & 0.43 \\
\hline EPA v. LA & -0.74 & 0.75 & -0.99 & 0.33 & $-2.24,0.77$ & \\
\hline DHA v. LA & $-1 \cdot 20$ & 0.71 & -1.69 & 0.10 & $-2.63,0.23$ & \\
\hline Trail-Making Task & & & & & & 0.32 \\
\hline EPA v. LA & 0.14 & 0.64 & 0.22 & 0.83 & $-1 \cdot 15,1 \cdot 43$ & \\
\hline DHA v. LA & 0.42 & 0.59 & 0.71 & 0.48 & $-0.77,1.61$ & \\
\hline Stroop score & & & & & & 0.53 \\
\hline EPA v. LA & -0.42 & 0.39 & -1.08 & 0.29 & $-1.21,0.37$ & \\
\hline DHA v. LA & -0.37 & 0.37 & -1.00 & 0.32 & $-1.13,0.38$ & \\
\hline Initial letter fluency & & & & & & 0.76 \\
\hline EPA $v$. LA & 1.07 & $2 \cdot 12$ & 0.51 & 0.62 & $-3.22,5.37$ & \\
\hline DHA v. LA & $4 \cdot 16$ & 1.99 & 2.09 & $0.04^{*}$ & $0.12,8.21$ & \\
\hline Excluded letter fluency & & & & & & 0.51 \\
\hline EPA v. LA & $-2 \cdot 26$ & 2.44 & -0.93 & 0.36 & $-7 \cdot 19,2 \cdot 66$ & \\
\hline DHA v. LA & -0.56 & $2 \cdot 31$ & -0.24 & 0.81 & $-5 \cdot 23,4 \cdot 11$ & \\
\hline
\end{tabular}

ICC, intra-class correlations; RAVLT, Rey Auditory Verbal Learning Test.

* Values were significantly different $(P<0.05)$

† Geriatric Depression Scale results shown with a statistical outlier removed from the placebo group.

‡ ICC gives the percentage of variance explained by individual subject differences; over $50 \%$ indicates reasonable goodness of fit. 


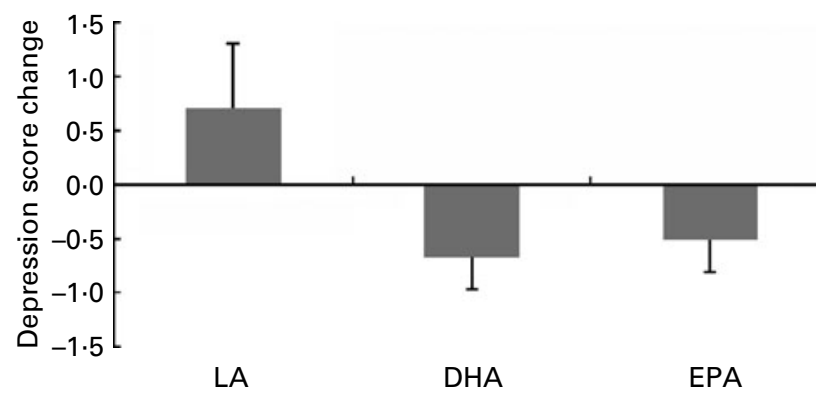

Fig. 2. Comparison of changes in depression scores in the treatment groups: DHA and EPA significantly different from linoleic acid (LA; $P=0.01, P=0.03$, respectively). Values are means, with standard deviations represented by vertical bars.

groups was not significantly different $(P=0 \cdot 39)$. Therefore, it was reasonable to assume that the data were missing at random at worst. The application of a linear multilevel model was appropriate for this missing pattern. All information could be used and predicted values could be obtained for all participants at each time point ${ }^{(42)}$. However, for the Memory Functioning Questionnaire, there were only twelve cases without missing data. Data imputation resulted in unacceptable data variability, so the Memory Functioning Questionnaire was not included in the analyses. Baseline comparisons between the groups showed no significant differences between the groups for outcome variables (data not shown). Treatment effects on outcome variables were assessed via visit (baseline and 6 months) $\times$ treatment interactions for the DHA and EPA groups compared with the LA group using linear mixed model analysis (Table 3).

\section{Depressive symptoms}

Following inspection of data distributions, one statistical outlier was removed from the placebo group in Brisbane as the GDS change score was $>3$ SD from the mean ${ }^{(43)}$ (nine at baseline and three at 6 months). According to Osborne \& Overbay $^{(44)}$, "the presence of outliers can lead to inflated error rates and substantial distortions of parameter and statistic estimates when using either parametric or nonparametric tests'. Following the removal of this outlier, there was a significant improvement in depression (GDS) scores in the EPA $(P=0.04)$ and DHA $(P=0.01)$ groups compared with the LA group (Fig. 2; Table 3). The amount of variance explained was $87 \%$, indicating a very good fit of the model to the data. The magnitude of change was slightly less than $0.5 \mathrm{SD}$ in the EPA group and greater than $0.5 \mathrm{SD}$ in the DHA group, which is accepted as a meaningful improvement in health-related quality of life ${ }^{(45)}$. Paired-samples $t$ tests found that within-group changes within each condition were only significant in the DHA group $(P=0.02)$.

\section{Quality of life}

There were no significant treatment effects on outcomes using mixed model analysis for physical or mental quality of life parameters (Table 3).

\section{Memory and cognitive function}

From assessments of memory and cognitive function, only Initial Letter Fluency scores significantly improved in the DHA treatment group compared with the LA group $(P=0 \cdot 04)$, explaining $76 \%$ of the variance and therefore a very acceptable goodness of fit (Fig. 3; Table 3). The Digits Backward score also showed improvement in the DHA group, although not statistically significant $(P=0 \cdot 10)$. The intra-class correlations in Table 3 indicate that the majority of outcome variables showed an acceptable fit of the data to the model and yet no other treatment effects.

\section{Associations between increased erythrocyte PUFA and changes in outcome variables}

Table 4 shows correlations between increased erythrocyte PUFA levels and improvements in outcome variables. Increased DHA plus EPA was correlated with improved depressive symptoms on the GDS $(P=0 \cdot 02)$. A reduced AA:EPA ratio was also associated with reduced depressive symptoms, further supporting the mixed model analysis results. Similarly, increased AA was associated with declines in self-reported mental health and a reduced AA:EPA ratio was correlated with improved self-reported mental health and vitality.

Increased DHA was significantly associated with improved self-reported physical functioning on the SF-36, and of those subscales, improved bodily pain was associated with improved depressive symptoms $(P<0.05)$; therefore, this may to some degree have contributed to improved mood.

\section{Baseline erythrocyte PUFA levels and improved outcome measures}

There were no significant correlations between baseline erythrocyte PUFA levels and improved outcome measures. However, comparisons between those with EPA plus DHA levels below and above the 50th percentile indicate that those with lower levels tended to show greater improvement in GDS scores $(P=0.066)$, physical functioning $(P=0.069)$, physical health $(P=0.088)$ and bodily pain $(P=0.058)$.

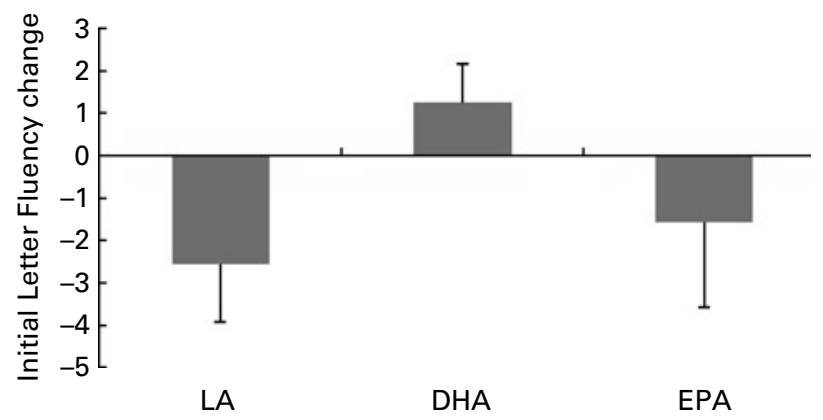

Fig. 3. Comparison of changes in Initial Letter Fluency: DHA significantly different from linoleic acid ( $L A ; P=0.04)$. 
Table 4. Correlations between change in PUFA and outcome variables from baseline to 6 monthst

\begin{tabular}{|c|c|c|c|c|c|c|c|c|c|c|c|c|c|c|c|c|c|c|c|c|c|c|c|}
\hline & EPA & DHA & $E+D$ & AA & $n-6$ & $A: E$ & GDS & RAV & $\mathrm{Rcl}$ & $\operatorname{Rec}$ & BNT & Strp & ILF & ELF & LNS & TM & DF & DB & PF & $\mathrm{PH}$ & $\mathrm{MH}$ & $\mathrm{BP}$ & Vit \\
\hline EPA & 1.00 & & & & & & & & & & & & & & & & & & & & & & \\
\hline DHA & -0.05 & 1.00 & & & & & & & & & & & & & & & & & & & & & \\
\hline$E+D$ & $0.54^{\star \star}$ & $0.81^{\star \star}$ & 1.00 & & & & & & & & & & & & & & & & & & & & \\
\hline $\mathrm{AA}$ & $-0.66^{\star \star}$ & $-0.37^{\star}$ & $-0.69^{\star \star}$ & 1.00 & & & & & & & & & & & & & & & & & & & \\
\hline$n-6$ & $-0.82^{* \star}$ & $-0.47^{\star \star}$ & $-0.87^{\star \star}$ & $0.81^{* \star}$ & 1.00 & & & & & & & & & & & & & & & & & & \\
\hline$A: E$ & $0.70^{\star *}$ & $0.42^{\star \star}$ & $0.76^{\star \star}$ & $-0.77^{\star \star}$ & $-0.84^{\star \star}$ & 1.00 & & & & & & & & & & & & & & & & & \\
\hline GDS & 0.23 & 0.30 & $0.39^{*}$ & -0.31 & -0.33 & $0.34^{*}$ & 1.00 & & & & & & & & & & & & & & & & \\
\hline RAV & -0.02 & -0.18 & -0.17 & -0.09 & 0.15 & -0.12 & 0.13 & 1.00 & & & & & & & & & & & & & & & \\
\hline $\mathrm{Rcl}$ & 0.14 & -0.02 & 0.07 & -0.01 & -0.09 & 0.03 & 0.27 & $0.39^{*}$ & 1.00 & & & & & & & & & & & & & & \\
\hline $\operatorname{Rec}$ & 0.04 & -0.17 & -0.13 & 0.15 & 0.06 & -0.26 & 0.04 & 0.08 & 0.28 & 1.00 & & & & & & & & & & & & & \\
\hline BNT & -0.19 & -0.14 & -0.22 & 0.15 & 0.23 & -0.17 & -0.20 & 0.08 & -0.08 & 0.08 & 1.00 & & & & & & & & & & & & \\
\hline Strp & 0.17 & 0.13 & 0.21 & -0.16 & -0.24 & 0.31 & 0.19 & 0.17 & 0.14 & -0.12 & -0.25 & 1.00 & & & & & & & & & & & \\
\hline $\mathrm{ILF}$ & -0.02 & 0.27 & 0.22 & -0.08 & -0.10 & 0.07 & -0.01 & 0.01 & 0.26 & 0.08 & $-0.34^{\star}$ & 0.04 & 1.00 & & & & & & & & & & \\
\hline ELF & -0.11 & 0.04 & -0.03 & 0.01 & 0.11 & -0.16 & 0.07 & 0.08 & -0.09 & 0.18 & -0.01 & -0.01 & -0.17 & 1.00 & & & & & & & & & \\
\hline LNS & -0.05 & -0.13 & -0.14 & 0.03 & 0.13 & 0.02 & -0.05 & 0.11 & 0.17 & -0.12 & 0.20 & 0.20 & 0.29 & $-0.47^{\star \star}$ & 1.00 & & & & & & & & \\
\hline $\mathrm{TM}$ & 0.20 & -0.10 & 0.03 & -0.09 & -0.04 & 0.08 & -0.16 & 0.07 & -0.23 & -0.16 & -0.10 & $0.44^{\star \star}$ & -0.27 & 0.24 & $-0.37^{*}$ & 1.00 & & & & & & & \\
\hline DF & -0.14 & 0.16 & 0.06 & 0.07 & 0.11 & -0.01 & -0.08 & -0.03 & 0.02 & -0.04 & -0.3 & 0.07 & $0.35^{*}$ & $-0.47^{\star \star}$ & 0.25 & 0.02 & 1.00 & & & & & & \\
\hline DB & -0.19 & -0.21 & -0.28 & 0.17 & 0.29 & -0.13 & $-0.40^{*}$ & 0.22 & -0.24 & -0.16 & 0.22 & 0.25 & -0.09 & -0.04 & 0.07 & 0.26 & 0.05 & 1.00 & & & & & \\
\hline $\mathrm{PF}$ & -0.02 & $0.37^{\star}$ & 0.30 & -0.07 & -0.11 & 0.21 & 0.25 & 0.19 & -0.08 & $-0.34^{*}$ & 0.07 & 0.26 & -0.03 & 0.01 & -0.18 & 0.23 & 0.21 & -0.05 & 1.00 & & & & \\
\hline PH & -0.15 & $0.39^{\star}$ & 0.25 & -0.06 & -0.01 & 0.21 & 0.30 & 0.21 & 0.13 & -0.13 & 0.11 & $0.35^{*}$ & 0.16 & 0.13 & -0.07 & 0.19 & 0.17 & 0.00 & $0.66^{*}$ & 1.00 & & & \\
\hline $\mathrm{MH}$ & 0.24 & 0.14 & 0.24 & $-0.37^{*}$ & -0.25 & $0.50^{\star *}$ & $0.37^{*}$ & 0.25 & 0.31 & 0.09 & -0.10 & 0.14 & 0.22 & -0.14 & -0.16 & -0.10 & 0.22 & -0.16 & 0.15 & $0.59^{\star \star}$ & 1.00 & & \\
\hline $\mathrm{BP}$ & 0.01 & $0.35^{*}$ & 0.29 & -0.04 & -0.10 & 0.30 & $0.33^{*}$ & 0.06 & 0.23 & -0.24 & -0.19 & $0.64^{\star \star}$ & 0.05 & 0.05 & -0.20 & 0.30 & 0.11 & 0.03 & $0.59^{\star \star}$ & ${ }^{*} 0.85^{\star \star}$ & $0.39^{\star}$ & 1.00 & \\
\hline Vit & 0.06 & 0.31 & 0.29 & -0.23 & -0.20 & $0.34^{*}$ & 0.27 & 0.29 & 0.29 & 0.03 & -0.05 & 0.11 & 0.18 & -0.05 & 0.03 & -0.00 & 0.13 & -0.09 & 0.29 & $0.65^{\star \star}$ & $0.79^{* \star}$ & ${ }^{*} 0.49^{* *}$ & 1.00 \\
\hline
\end{tabular}

E + D, EPA + DHA; AA, arachidonic acid; $n-6$, total $n-6$ PUFA; A:E, AA:EPA ratio; GDS, Geriatric Depression Scale; RAV, Rey Auditory Verbal Learning Test, total recall; Rcl, Rey Auditory Verbal Learning Test, delayed recall; Rec, Rey Auditory Verbal Learning Test, recognition; BNT, Boston Naming Task; Strp, Stroop Colour-Word Test; ILF, Initial Letter Fluency; ELF, Excluded Letter Fluency; LNS, Letter-Number Sequencing; TM, Trail Making; DF, Digits Forward; DB, Digits Backward; PF, physical functioning; PH, physical health; MH, mental health; BP, bodily pain; Vit, vitality.

${ }^{\star} P<0.05,{ }^{* *} P<0.01$.

† All change variables are calculated so that any change in a positive direction indicates a positive change: i.e. for PUFA, an increase in PUFA levels; for AA:EPA, a decreased ratio; for all other variables, an improvement in the outcome (e.g. positive change in GDS scores, reduction in depressive symptoms; positive change in bodily pain, reduction in bodily pain, etc.). 


\section{Discussion}

We investigated the effects of 6 months' supplementation with fish oils rich in either EPA or DHA $v$. a LA-rich control (safflower oil) on memory and executive function, depressive symptoms and health-related quality of life in elderly people with MCI. A third of the sample had scores that indicated possible depression, significantly greater than a healthy control group recruited at baseline ${ }^{(46)}$, and they were equally assigned to each treatment condition. The treatment was well tolerated overall, with no adverse effects in the DHA group and a handful of gastrointestinal complaints in each of the LA and EPA groups.

Depressive symptom scores were significantly reduced after 6 months of high-EPA and high-DHA supplementation compared with LA and increased erythrocyte levels of EPA plus DHA were associated with improved depressive symptoms, as was a reduced ratio of AA:EPA. Increased erythrocyte DHA levels were associated with reduced bodily pain, and, although not significantly, with other items associated with physical health. Therefore, reduced depressive symptoms may to a small degree have been accounted for by reduced bodily pain resulting from improved physical functioning with DHA supplementation. Although depressive symptoms can increase the risk of developing dementia in elderly people with cognitive impairment, to our knowledge, this has not been addressed in previous studies.

Research has independently indicated that $n$-3 PUFA supplementation may assist with both cognitive impairment and depression, and it is possible that combined depression and cognitive impairment is indicative of common underlying biological mechanisms that may in some cases be attributed to suboptimal n-3 PUFA levels ${ }^{(47)}$. To our knowledge, other studies have also not yet reported comparisons of high-EPA and high-DHA supplementation for depressive symptoms or cognitive impairment. Although high EPA or ethyl-EPA has been reportedly effective in some studies with depression $^{(48,49)}$, we cannot rule out the effect of DHA. For instance, most studies have not correlated increased erythrocyte PUFA levels with outcomes. In one study that used a supplement containing DHA plus EPA, significant correlations between increased DHA and improved depressive symptoms were found ${ }^{(50)}$ (although this study had a large placebo effect as both groups received counselling, masking differences between groups) ${ }^{(51)}$. Another study that used a supplement with an EPA:DHA ratio of 2:1 reported baseline levels of erythrocyte DHA at $2.4 \%$ and this increased to $5 \cdot 8 \%{ }^{(52)}$; therefore, it is possible that DHA contributed to the positive findings. Other methodological considerations include the fact that one of the pure DHA studies was also the shortest study on $n$-3 PUFA and depression (6 weeks) ${ }^{(53)}$. Furthermore, only three studies in depression have used DHA compared with ten or more that have focused on $\mathrm{EPA}^{(15)}$. The present study indicates that DHA may be equally, if not more, effective than EPA for improving mood. This needs to be investigated further in clinical depression. Given the relative ratios of EPA and DHA in brain tissue, it may be that EPA is not required, nor effective, in as large proportions as DHA.
Of the cognitive outcomes, significant improvements were only detected in the DHA group for Initial Letter Fluency, a test of fluid thinking ability. Scores on Digits Backward also showed trends for improvement in the DHA group. These tests measure executive function, which is impaired in dementia. Increased DHA was correlated with improved Initial Letter Fluency, although not statistically significant. It should be noted that our sample had baseline EPA plus DHA levels over $5 \%$ of erythrocyte fatty acids, whereas Chiu et al. ${ }^{(18)}$ had an average combined level of $4 \cdot 2 \%$. Therefore, it is possible that our MCI sample had higher levels than those that have derived cognitive benefits from $n-3$ PUFA. This may also be attributed in part to the small sample size, the outcome measures and/or a lower $n-6: n-3$ ratio in our population. Our final numbers in each group were smaller than the numbers in MCI subgroups from previous studies that detected reduced cognitive decline with $2 \mathrm{~g} n$-3 PUFA over 6 months $^{(18,19)}$. There is also variation in an individual's performance on cognition from day to day, and it is possible, as discussed previously $^{(15)}$ that the failure of this and many other studies that employ a range of cognitive tests to measure outcomes is due to this daily variation being larger than nutritional treatment effects, thereby potentially rendering the tests largely insensitive to such effects.

In conclusion, these results indicate that DHA-rich and EPArich fish oils may be effective for depressive symptoms and health parameters, exerting variable effects on cognitive and physical outcomes. Reducing n-6 PUFA intake as well as increasing $n$-3 PUFA intake for a more balanced ratio may be beneficial, and this should also be explored further. It is possible that reductions in mood and verbal fluency in the LA group represented a normal decline in this population, although we did observe significant correlations between a decreased ratio of AA:EPA and improved mood. The present findings suggest that pure EPA supplements employed in some mental health studies may not be the optimal choice. Future research should further investigate this in larger, clinically depressed samples of people with MCI and conduct longitudinal follow-up to assess whether the risk of progression to dementia is reduced in these populations. Research with $n$-3 PUFA and mental health in elderly populations should also factor in favourable influences of fish oil on physical function, particularly joint pain, which could also have an impact on mood.

\section{Acknowledgements}

This study was funded by an Australian Research Council Linkage grant in partnership with Novasel Australia. The authors acknowledge the assistance of all volunteers in the present study, plus the support of the Australian Centre for Metabolic Fitness, and Professors Andrew Hills and Karen Sullivan at the Queensland University of Technology. N. S. and P. R. C. H. designed the research; N. S., C. M. M., J. D. B., A. M. C. and S. J. S. conducted the research; J. P., N. S., C. M. M. and P. R. C. H. analysed the data; N. S. prepared the manuscript. All authors read, edited and approved the final manuscript. We declare no conflicts of interest. 


\section{References}

1. Blennow K, de Leon MJ \& Zetterberg H (2006) Alzheimer's disease. Lancet 368, 387-403.

2. Ferri CP, Prince M, Brayne C, et al. (2005) Global prevalence of dementia: a Delphi consensus study. Lancet 366 , 2112-2117.

3. Gauthier S, Reisberg B, Zaudig M, et al. (2006) Mild cognitive impairment. Lancet 367, 1262-1270.

4. Chertkow H (2002) Mild cognitive impairment. Curr Opin Neurol 15, 401-407.

5. Gabryelewicz T, Styczynska M, Pfeffer A, et al. (2004) Prevalence of major and minor depression in elderly persons with mild cognitive impairment - MADRS factor analysis. Int J Geriatr Psychiatry 19, 1168-1172.

6. Modrego P \& Ferrández J (2004) Depression in patients with mild cognitive impairment increases the risk of developing dementia of Alzheimer type. Arch Neurol 61, 1290-1293.

7. Simopoulos AP (1991) Omega-3 fatty acids in health and disease and in growth and development. Am J Clin Nutr 54, 438-463.

8. Assisi A, Banzi R, Buoinocore C, et al. (2006) Fish oil and mental health: the role of $n-3$ long-chain polyunsaturated fatty acids in cognitive development and neurological disorders. Int Clin Psychopharmacol 21, 319-336.

9. Chalon S (2006) Omega-3 fatty acids and monoamine neurotransmission. Prostaglandins Leukot Essent Fatty Acids $\mathbf{7 5}$ $259-269$.

10. Haag M (2003) Essential fatty acids and the brain. Can $J$ Psychiatry 48, 195-203.

11. Hibbeln JR, Ferguson TA \& Blasbalg TL (2006) Omega-3 fatty acid deficiencies in neurodevelopment, aggression and autonomic dysregulation: opportunities for intervention. Int Rev Psychiatry 18, 107-118.

12. Simopoulos AP (1999) Essential fatty acids in health and chronic disease. Am J Clin Nutr 70, Suppl. 3, 560S-569S.

13. Yehuda S, Rabinovitz S, Carasso RL, et al. (2002) The role of polyunsaturated fatty acids in restoring the aging neuronal membrane. Neurobiol Aging 23, 843-853.

14. Söderberg M, Edlund C, Kristensson K, et al. (1991) Fatty acid composition of brain phospholipids in aging and in Alzheimer's Disease. Lipids 26, 421-425.

15. Sinn N, Milte C \& Howe PRC (2010) Oiling the brain: a review of randomised controlled trials of omega-3 fatty acids in psychopathology across the lifespan. Nutrients $\mathbf{2}$, $128-170$.

16. Terano T, Fujishiro S, Ban T, et al. (1999) Docosahexaenoic acid supplementation improves the moderately severe dementia from thrombotic cerebrovascular diseases. Lipids 34, Suppl., S345-S346.

17. Yehuda S, Rabinovitz S, Carasso RL, et al. (1996) Essential fatty acids preparation (SR-3) improves Alzheimer's patients quality of life. Int J Neurosci 87, 141-149.

18. Chiu C-C, Su K-P, Cheng T-C, et al. (2008) The effects of omega-3 fatty acids monotherapy in Alzheimer's disease and mild cognitive impairment: a preliminary randomized double-blind placebo-controlled study. Prog Neuro-Psychopharmacol Biol Psychiatry 32, 1538-1544.

19. Freund-Levi Y, Basun H, Cederholm T, et al. (2008) Omega-3 supplementation in mild to moderate Alzheimer's disease: effects on neuropsychiatric symptoms. Int $J$ Geriatr Psychiatry 23, 161-169.

20. Kotani S, Sakaguchi E, Warashina S, et al. (2006) Dietary supplementation of arachidonic and docosahexaenoic acids improves cognitive dysfunction. Neurosci Res 56, $159-164$
21. Dangour AD, Allen E, Elbourne D, et al. (2010) Effect of 2-y $n-3$ long-chain polyunsaturated fatty acid supplementation on cognitive function in older people: a randomized, double-bind, controlled trial. Am J Clin Nutr 91, 1725-1732.

22. van de Rest O, Geleijnse JM, Kok FJ, et al. (2008) Effect of fish oil on cognitive performance in older subjects: a randomized, controlled trial. Neurology 71, 430-438.

23. Yurko-Mauro K (2010) Cognitive and cardiovascular benefits of docosahexaenoic acid in aging and cognitive decline. Curr Alzheimer Res 7, 190-196.

24. Sinclair AJ, Begg D, Mathai M, et al. (2007) Omega 3 fatty acids and the brain: review of studies in depression. Asia Pac J Clin Nutr 16, Suppl. 1, 391-397.

25. Winblad B, Palmer K, Kivipelto M, et al. (2004) Mild cognitive impairment - beyond controversies, towards a consensus: report of the International Working Group on Mild Cognitive Impairment. J Int Med 256, 240-246.

26. Wechsler D (1997) Wechsler Memory Scale-III. Administration and Scoring Manual. San Antonio, TX: Psychological Corporation.

27. Kalbe E, Kessler J, Calabrese P, et al. (2004) DemTect: a new, sensitive cognitive screening test to support the diagnosis of mild cognitive impairment and early dementia. Int J Geriatr Psychiatry 19, 136-143.

28. Altman DG \& Bland JM (2005) Treatment allocation by minimisation. Br Med J 330, 843.

29. Sheikh JI \& Yesavage JA (1986) Geriatric Depression Scale (GDS): recent evidence and development of a shorter version. In Clinical Gerontology: A Guide to Assessment and Intervention, pp. 165-173. New York: The Haworth Press.

30. Sanson-Fisher RW \& Perkins JJ (1998) Adaptation and validation of the SF-36 Health Survey for use in Australia. J Clin Epidemiol 51, 961-967.

31. Raschetti R, Albanese E, Vanacore N, et al. (2007) Cholinesterase inhibitors in mild cognitive impairment: a systematic review of RCTs. Pub Libr Science: Med 4, 1818-1828.

32. Gilewski MJ \& Zelinski EM (1988) Memory Functioning Questionnaire. Psychopharmacol Bull 24, 665-670.

33. Lezak MD (1995) Neuropsychological Assessment, 3rd ed. New York: Oxford University Press.

34. Wechsler D (1997) Wechsler Adult Intelligence Scale, 3rd ed. San Antonio, TX: Psychological Corporation.

35. Kent P, Bryan J \& Clark M (2004) Preclinical signs of dementia in a community sample: a longitudinal analysis. Brain Impairment 5, 108.

36. Sachs TL, Clark CR, Pols RG, et al. (1991) Comparability and stability of performance of six alternative forms of the Dodrill-Stroop Color-Word Test. Clin Neuropsychol 54, 1063-1070.

37. Bryan J, Luszcz M \& Crawford JR (1997) Verbal knowledge and speed of information processing as mediators of age differences in verbal fluency performance among older adults. Psychol Aging 12, 473-478.

38. Rosen WG (1980) Verbal fluency in aging and dementia. J Clin Exp Neuropsychol 2, 135-146.

39. Bligh EG \& Dyer WJ (1959) A rapid method of total lipid extraction and purification. Can J Physiol Pharmacol 37, 911-917.

40. Folch J, Lees M \& Sloane Stanley GH (1957) A simple method for the isolation and purification of total lipides from animal tissues. J Biol Chem 226, 497-509.

41. Lepage G \& Roy CC (1986) Direct transesterification of all classes of lipids in a one-step reaction. J Lipid Res $\mathbf{2 7}$, 114-120.

42. Hedeker D \& Gibbons RD (2006) Longitudinal Data Analysis. New Jersey: John Wiley \& Sons Inc. 
43. Tabachick B \& Fidell L (2001) Using Multivariate Statistics. Needham Heights, MA: Allyn \& Bacon.

44. Osborne JW \& Overbay A (2004) The power of outliers (and why researchers should always check for them). Pract Assess Res Eval 9. (Retrieved 25 May 2011 from http://PAREonline. net/getvn.asp? $v=9 \& n=6$ ).

45. Norman GR, Sloan JA \& Wyrwich KW (2003) Interpretation of changes in health-related quality of life: the remarkable universality of half a standard deviation. Med Care $\mathbf{4 1}$, $582-592$.

46. Milte C, Sinn N, Street SJ, et al. (2011) Erythrocyte polyunsaturated fatty acid status, memory, cognition and mood in older adults with mild cognitive impairment and healthy controls. Prostaglandins Leukot Essent Fatty Acids 84, $153-161$.

47. Sinn N \& Howe PRC (2008) Mental health benefits of omega-3 fatty acids may be mediated by improvements in cerebral vascular function. Biosci Hypotheses 1, 103-108.

48. Nemets B, Stahl Z \& Belmaker RH (2002) Addition of omega-3 fatty acid to maintenance medication treatment for recurrent unipolar depressive disorder. Am J Psychiatry 159, 477-479.
49. Peet M \& Horrobin DF (2002) A dose-ranging study of the effects of ethyl-eicosapentaenoate in patients with ongoing depression despite apparently adequate treatment with standard drugs. Arch Gen Psychiatry 59, 913-919.

50. Meyer B, Grenyer BFS, Crowe $\mathrm{T}$ et al. (editors) (2004) Improvement of major depression is associated with increased erythrocyte DHA - subset analysis in a doubleblind omega-3 supplementation trial. International Society for the Study of Fatty Acids and Lipids (ISSFAL), 27 June-1 July, Brighton, UK.

51. Grenyer BFS, Crowe T, Meyer B, et al. (2007) Fish oil supplementation in the treatment of major depression: a randomised double-blind placebo-controlled trial. Prog Neuropsychopharmacol Biol Psychiatry 31, 1393-1396.

52. Su K-P, Huang S-Y, Chiu C-C, et al. (2003) Omega-3 fatty acids in major depressive disorder: a preliminary doubleblind, placebo-controlled trial. Eur Neuropsychopharmacol 13, 267-271.

53. Marangell LB, Martinez JM, Zboyan HA, et al. (2003) A double-blind, placebo-controlled study of the omega- 3 fatty acid docosahexaenoic acid in the treatment of major depression. Am J Psychiatry 160, 996-998. 